\title{
8 \\ SHINING LIGHT ON HUMAN IMMUNITY
}

ROBYN LUCAS, ASHWIN SWAMINATHAN AND KEITH DEAR

\section{Abstract}

The immune system is a key interface between environmental exposures and human health. It provides protection against infectious agents and toxins reaching the skin and mucosal surfaces and maintains internal surveillance to destroy abnormal cells. Overactivity of the immune system can result in autoimmune diseases, such as multiple sclerosis, while medicine takes advantage of immune processes to stimulate protection from pathogens, through vaccination. Ultraviolet radiation from the sun can suppress the immune response, potentially decreasing the risk of autoimmune disease development but impairing the immune response to vaccination. This chapter uses these two examples, multiple sclerosis and vaccination, to illustrate a pathway of research that began with observation of variations in disease or vaccination outcomes and proceeded through epidemiological and biomedical studies of increasing depth and sophistication. The end point - yet to be achieved but within sight - will be the translation of the findings into actions to improve public health. Tony McMichael's key contributions to each step of this research pathway are described.

\section{Introduction}

In the 1920s, researchers observed that multiple sclerosis, a debilitating degenerative neurological disease, was more common in regions at higher latitude. This finding, replicated in both the northern and southern hemispheres, is an unusual but important feature of the epidemiology of multiple sclerosis. It suggests that environmental factors may play a role in its aetiology. Recent 
work indicating that the incidence of multiple sclerosis has increased over the past 30 years, after accounting for improved diagnosis, also indicates a role for changing environmental determinants.

A key feature of Tony McMichael's work was to discern and quantify the links between human health and the environment, and the changing ways in which humans interacted with that environment. One facet of that work involved exploring the link between immune function and exposure to ultraviolet (UV) radiation at the population level, particularly in relation to the risk of autoimmune diseases. This chapter begins with a brief sketch of the human immune system, followed by a story of translational public health research: from observation to investigation to testing; and hopefully to prevention.

\section{A Brief Introduction to the Human Immune System}

The immune system protects against attack from foreign organisms and maintains internal surveillance to detect and destroy abnormal cells. Humans have both innate and adaptive immune systems, with considerable cross-communication between them. Innate immunity is found in all plants and animals. It provides an immediately maximal but non-specific immune response to injury or pathogen exposure. There is no immunological memory - that is, the innate response is the same each time the system is activated. In contrast, the adaptive immune system is found only in higher animals, specifically, jawed vertebrates. There is a lag of hours or days between pathogen exposure and maximal immune response, but the response is pathogen or antigen specific, and a subsequent exposure to the same pathogen results in a more immediate, targeted physiological attack. This is immunological memory, the basis of vaccination.

The adaptive immune response has two arms that act in concert. Humoral immunity involves the production of antibodies, macromolecules that coat the invading/foreign pathogen and facilitate its destruction. Cell-mediated immunity relies on specialised lymphocytes to recognise and destroy pathogens. Both systems require cells that present antigens (antigen-presenting cells, e.g. dendritic cells) to lymphocytes, including B-lymphocytes and various types of T-lymphocytes (e.g. T-helper cells, T-cytotoxic cells, T-regulatory cells). Different types of cells secrete specific chemicals (cytokines) that permit 'communication' across the immune system during an immunological response. 


\section{Multiple Sclerosis and Sun Exposure: From Geography to Prevention}

Multiple sclerosis (MS) is an inflammatory disease of the central nervous system, usually leading to progressive deterioration of nerve function. It is the commonest disabling neurological disorder of young adults. MS is thought to be an autoimmune disease, caused by an immune-mediated attack of cells of the adaptive immune system on the myelin-producing cells of the central nervous system, the oligodendrocytes. Myelin is a lipoprotein that coats and insulates nerve fibres, nourishing them and increasing the speed of conduction of nerve impulses. Disruption of myelin results in slowing of conduction and, over time, degeneration of nerve fibres. People with MS generally suffer recurrent bouts of neurological dysfunction, manifesting as weakness, localised altered sensation or visual impairment, eventually followed by cumulative neurological deficits and increasing disability.

\section{Risk Factors for MS}

Relatives of a person with MS are also more likely to develop the disease, with the risk increasing according to the closeness of the genetic relationship: for example, the risk in full siblings is approximately 35 times that of the general population, and in monozygotic twins approximately 270 times greater (Ebers, 2008). Despite these high relative risks, with an absolute incidence generally of the order of five to 10 new cases per 100,000 population per year, the absolute risk of MS is low, even in close family members. A growing number of susceptibility genes are recognised, but each of these only slightly increases the risk.

Several lines of evidence indicate the importance of environmental exposures in the pathogenesis of MS, particularly observations of increasing incidence over recent decades and the strong latitudinal gradient seen for incidence, prevalence and mortality. Possible contenders that would account for some of these findings include infections, for example Epstein-Barr virus, canine distemper virus, human herpes virus 6 and others, diet and changing intestinal microbiomes and exposure to the sun or related factors. This story focuses on sun exposure, without discounting the possible importance of other factors that may act independently or interactively.

A positive latitude gradient in MS was first noted by Davenport in 1922, based on data from conscripts to World War I (Davenport, 1922). In 1960, Acheson et al. further explored this finding in an analysis of the place of birth of soldiers in World War II who developed MS. Here, there was a strong correlation 
between MS prevalence and latitude of birthplace $(r=0.76)$, and a slightly stronger (inverse) correlation with average daily December solar UV radiation $(r=-0.80)$ (Acheson and Bachrach, 1960; Acheson et al., 1960). A number of other studies over ensuing years have also demonstrated a latitude gradient in several different countries, including Australia, where the prevalence of MS in 1981 was almost seven times higher in Tasmania than in northern Queensland (McLeod et al., 1994).

These ecological studies prompted individual-level observational studies, but the evidence from case-control studies of MS patients and healthy controls was contradictory, variously showing an increase, decrease, or no change in risk in association with self-reported time outdoors.

\section{The Rise of Photoimmunology}

The 1970s saw the development of interest in the immune effects of exposure to the sun - photoimmunology. Kripke's work showed that immunosuppression following exposure of the skin to UV radiation contributed to skin cancer development and diminished host resistance to infectious diseases in animals (Kripke, 1994). UV irradiation of the skin was shown to trigger a cascade of immunological events, including decreased antigen-presenting activity, release of the cytokine IL-10 and other immune mediators, and induction of regulatory T-cell activity. The net result was suppression of antigen-specific adaptive immunity, both locally (at the site of immunological challenge) and systemically. In addition, there was a shifting of the balance in T-cell activity towards a suppressive milieu.

\section{Bringing It All Together}

Exposure to the UV-B wavelengths of solar radiation $(280-315 \mathrm{~nm})$ is the source of over 90 per cent of vitamin D stores in people living in most regions of the world. Accordingly, by 1991, animal studies had tested the effect of administering the active form of vitamin $\mathrm{D}$ and shown that high doses completely prevented the development of the animal model of MS, experimental allergic encephalomyelitis (Lemire and Archer, 1991).

By the 1990s, two hypotheses on the causes of MS were in the spotlight: the vitamin D hypothesis that insufficiency leads to an overactive immune system, and an infection hypothesis that a viral infection somehow initiates the autoimmune process. Both hypotheses have been widely tested over the ensuing years. Many case-control and cohort studies have now provided evidence that 
suggests that lower vitamin D status and a past history of symptomatic infection with Epstein-Barr virus (EBV, glandular fever or infectious mononucleosis) are associated with increased risk of MS.

In 1994, Tony McMichael moved to the London School of Hygiene and Tropical Medicine (LSH\&TM), where Sir Donald Acheson was Professor of International Health. Tony was aware of, and intrigued by, Acheson's earlier papers, and the two discussed them. During this period, Tony reviewed the health chapter for the 1994 report of the Environmental Effects Assessment Panel for the Montreal Protocol on Ozone Depletion (Longstreth et al., 1994). This included a review of Kripke's recent work on the immune suppression that occurred following UV irradiation and its importance for skin cancer development. Tony and a colleague at the LSH\&TM, Andy Hall, proposed an integrated hypothesis, bringing together this new work with the environmental patterns and the infection hypothesis:

We therefore propose that UVR-induced suppression of immune function, maximal at low latitudes, attenuates the autoimmune process that underlies MS. This attenuation could occur either by a diminution in the initial sensitization of T-cells to a newly encountered (and myelin basic protein-'mimicking') viral antigen, or by a reduced intensity of cell-mediated immune response to ongoing infection with the virus. (McMichael and Hall, 1997)

This paper was the first to link the apparent protective effect of UV radiation with evidence of risk associated with viral infection. It suggested that UV radiation played a larger role in the MS story than that through vitamin D only, and that viral infection in the presence of impaired environmental immune suppression might be important. It provided a number of testable predictions from the hypothesis, of which some have been supported by empirical evidence, albeit to a limited extent. For example, they hypothesised that 'MS incidence rates (at least in fair-skinned populations) will decline in response to any further increase in population exposure to UVR'. Instead, in many fair-skinned populations, the prevailing public health advice has been to decrease exposure to UV radiation to control skin cancer risk. It has been proposed that this may explain the loss of the latitude gradient in MS incidence between successive waves of the Nurses' Health Study in the USA: higher levels of sun protection in the sunnier south may explain greater increases in MS incidence in the south than in the north (Ascherio and Munger, 2007).

In 2003, on his return to Australia, McMichael led the Ausimmune Study that examined sun exposure, vitamin $\mathrm{D}$ and infection history in people with a first clinical diagnosis of central nervous system (CNS) demyelination (Lucas et al., 2007), prior to a diagnosis of MS. A weakness of studies of vitamin D levels in 
people with frank MS is the possibility of reverse causation, whereby people with MS have lower vitamin D status because they have MS, rather than the other way around. This is likely because the disease causes heat intolerance, so that patients tend to avoid the sun, and because the physical disability that develops in later stages makes outdoor exercise difficult. By recruiting people with the first symptoms of an illness that may progress to MS, the Ausimmune Study avoided this possibility. The study was able to examine a wide range of genetic and environmental factors, including infections, and the interactions between them. Key findings of the study were that there was preservation of a latitude gradient of approximately the same magnitude for incidence as had been previously described for prevalence in 1981 data (Taylor et al., 2010); and that lower vitamin D status (Lucas et al., 2011b), past history of infectious mononucleosis and higher levels of EBV antibodies (Lucas et al., 2011a) were all independently associated with increased risk of being a case with a first diagnosis of CNS demyelination. In addition, sun exposure itself, over the life course or the previous three years, was associated with a lower risk of being a case, even after adjustment for other known risk factors.

Based on the weight of accumulating evidence, including that from the Ausimmune Study, a vitamin D prevention trial was initiated in Australia in 2012. The PrevANZ Study will randomly allocate adult participants with a first diagnosis of CNS demyelination to 1,000, 5,000 or 10,000 IU (international units) of vitamin D3 per day, or to placebo (i.e. zero IU), to test whether vitamin D can prevent progression to MS. Importantly, the study will include detailed measurements of sun exposure to distinguish any independent protective effects of sun exposure and vitamin D. Data from questionnaires and blood samples will provide information on past infections, to test integrative hypotheses further, including the one proposed by McMichael and Hall.

Careful observation of disease patterns in ecological studies stimulated the formation of hypotheses about sun exposure, vitamin D and infection, which led to individual-level observational studies to test these hypotheses. Positive findings in relation to vitamin $\mathrm{D}$ and sun exposure have further led to clinical trials to test whether observed associations are causal. It has been estimated that if there is a causal association between vitamin D insufficiency and MS incidence, then at least 40 per cent of cases of MS could be prevented (Ascherio and Munger, 2007). 


\section{Not Only Benefits}

In consideration of human health and the environment, McMichael wrote about the changing nature of the interaction between humans and their environments (McMichael, 2001). Concurrent risks and benefits of environmental exposures for human health, and levels of optimal exposure, are typical of natural selection and survival of the fittest. So, too, for sun exposure. While higher levels of sun exposure may be protective against MS, either independently via vitamin D production or through interaction with viral infection, higher levels also increase the risk of skin cancers and eye diseases. The immunosuppressive effects, potentially beneficial for MS, may impair the immune response to vaccination - a factor that could have considerable importance for those regions at low latitude but with high microbial load. McMichael's research interest in this field continued to explore such risks, through the recently completed Australian Ultraviolet Radiation and Immunity (AusUVI) Study.

\section{Exposure to UV Radiation and Vaccination}

Vaccination against common communicable diseases has been one of the most successful public health measures of all time. Any process that might compromise vaccine effectiveness is therefore of profound importance to public health and warrants rigorous scientific investigation. Numerous studies have shown an association between reduced vaccine effectiveness and its administration in high compared with low ambient UV environments (i.e. summer versus winter months and/or low- versus high-latitude regions) (Norval and Woods, 2011). However, these are weak surrogates of an individual's personal sun exposure; that is influenced by a wide range of factors such as how much time they spend outdoors, whether they use sun protection and what types of clothing are worn. Using ecological proxies risks making false inferences about the exposureoutcome relationship (the so-called 'ecological fallacy').

The AusUVI Study, of which Professor McMichael was a key investigator, was conducted to determine the influence of exposure to solar UV radiation, attained through activities of normal daily life, on the primary immune response to subcutaneous vaccination with an experimental vaccine antigen, keyhole limpet haemocyanin (KLH). The advantages of this prospective, two-centre observational study included direct measurement of exposure to UV radiation at an individual level, quantification of potential confounding immune-modulatory factors (e.g. physical fitness, age, psychological well-being) and sophisticated measurement of an array of vaccine-associated immune outcomes. 
The results of the AusUVI Study have only recently become available. They show that higher personal exposure to UV radiation in the peri-vaccination period did, indeed, suppress the antigen (KLH)-specific cell-mediated immune response. Exposure to UV radiation in the day prior to vaccination was most influential. Other findings, of a change in the proportion of a circulating subpopulation of T-helper lymphocytes, may have relevance to the pathogenesis of autoimmune diseases (Veldhoen et al., 2008) and the association between sun exposure and autoimmune diseases, such as MS. Interestingly, here, vitamin D status was not associated with immune outcomes, i.e. this was a sun exposure effect rather than a vitamin $\mathrm{D}$ effect.

The clinical implications of the findings of the AusUVI Study and related studies are not presently clear. Using immune parameters as a surrogate for increased disease risk (or reduced vaccine effectiveness) is problematic, particularly when those changes are subtle, because the human immune system is endowed with multiple redundancies and functional reserve capacity. However, at a population level (which includes aged, very young and ill individuals), less profound impairment of specific immune processes occurring with high prevalence may manifest as an increased incidence of common infections (e.g. influenza, rhinovirus, otitis media) and/or reduced vaccine effectiveness.

Further well-designed studies using commonly administered vaccines (e.g. measles, pneumococcal) need to be performed where, among other considerations, exposure to UV radiation at an individual level is quantified, confounding variables are accounted for and clinical outcomes (e.g. vaccine effectiveness against disease) measured. Conducting these studies in vulnerable populations may be additionally revealing - e.g. assessing the influence of exposure to solar UV radiation on childhood vaccinations in peri-equatorial regions. If commercial vaccine effectiveness is indeed shown to be compromised by high individual UV radiation exposure, potential public health strategies would include the promotion of sun-protective clothing and sunscreen at around the time of vaccine administration.

\section{Conclusion}

McMichael's chief contribution to this story of public health translation was through the bringing together of separate lines of research to generate testable hypotheses, and then exploring predictions from those hypotheses in observational studies. His key insight was that, even in an intensely biomedical field like immunology, untangling disease aetiology could benefit hugely from complementing the laboratory work with epidemiological research undertaken from a global population-level perspective. The work has real potential to 
decrease the incidence of a disabling, devastating neurological disease, as well as to shed light on new risks related to UV-induced immune suppression that will need to be considered in vaccination delivery.

\section{References}

Acheson, E.D. \& Bachrach, C.A. 1960. The distribution of multiple sclerosis in U.S. veterans by birthplace. The American Journal of Hygiene 72, 88-99.

Acheson, E.D., Bachrach, C.A. \& Wright, F.M. 1960. Some comments on the relationship of the distribution of Multiple Sclerosis to latitude, solar radiation and other variables. Acta Psychiatrica Scandinavica 35, 132-47.

Ascherio, A. \& Munger, K.L. 2007. Environmental risk factors for multiple sclerosis. Part II: Noninfectious factors. Annals of Neurology 61, 504-13.

Davenport, C. 1922. Multiple sclerosis from the standpoint of geographic distribution and race. Archives of Neurology and Psychiatry 8, 51-8.

Ebers, G.C. 2008. Environmental factors and multiple sclerosis. Lancet Neurology 7, 268-77.

Kripke, M.L. 1994. Ultraviolet radiation and immunology: something new under the sun - presidential address. Cancer Research 54, 6102-5.

Lemire, J.M. \& Archer, D.C. 1991. 1,25-dihydroxyvitamin D3 prevents the in vivo induction of murine experimental autoimmune encephalomyelitis. Journal of Clinical Investigation 87, 1103-7.

Longstreth, J., de Gruijl, F.R., Kripke, M.L., Takizawa, Y. \& van der Leun, J.C. 1994. Effects of increased ultraviolet radiation on human health. In: UNEP (ed.) Environmental Effects of Ozone Depletion, 1994 Assessment. UNEP, Nairobi, Kenya, 23-48.

Lucas, R.M., Ponsonby, A.L., McMichael, A.J., van der Mei, I., Chapman, C., Coulthard, A., et al. 2007. Observational analytic studies in multiple sclerosis: controlling bias through study design and conduct. The Australian Multicentre Study of Environment and Immune Function. Multiple Sclerosis Journal 13, 827-39.

Lucas, R.M., Ponsonby, A.L., Dear, K., Valery, P.C., Pender, M.P., Taylor, B.V., et al. 2011a. Sun exposure and vitamin D are independent risk factors for CNS demyelination. Neurology 76, 540-548. 
Lucas, R.M., Ponsonby, A.L., Dear, K., Valery, P.C., Pender, M.P., J.M. Burrows, et al. 2011b. Current and past Epstein-Barr virus infection in risk of initial CNS demyelination. Neurology 77, 371-9.

McLeod, J.G., Hammond, S.R. \& Hallpike, J.F. 1994. Epidemiology of multiple sclerosis in Australia. With NSW and SA survey results. Medical Journal of Australia 160, 117-22.

McMichael, A.J. 2001. Human Frontiers, Environments and Disease: Past Patterns, Uncertain Futures. Cambridge University Press, Cambridge, UK.

McMichael, A.J. \& Hall, A.J. 1997. Does immunosuppressive ultraviolet radiation explain the latitude gradient for multiple sclerosis? Epidemiology 8, 642-5.

Norval, M. \& Woods, G.M. 2011. UV-induced immunosuppression and the efficacy of vaccination. Photochemical \& Photobiological Sciences 10, 1267-74.

Taylor, B.V., Lucas, R.M., Dear, K., Kilpatrick, T.J., Pender, M.P., van der Mei, I.A., et al. 2010. Latitudinal variation in incidence and type of first central nervous system demyelinating events. Multiple Sclerosis Journal 16, 398-405.

Veldhoen, M., Hirota, K., Westendorf, A.M., Buer, J., Dumoutier, L., Renauld, J.-C., et al. 2008. The aryl hydrocarbon receptor links TH17-cell-mediated autoimmunity to environmental toxins. Nature 453, 106-9. 
This text is taken from Health of People, Places And Planet:

Reflections based on Tony McMichael's four decades of contribution to epidemiological understanding, edited by Colin D. Butler, Jane Dixon and Anthony G. Capon, published 2015 by ANU Press, The Australian National University, Canberra, Australia. 